\title{
Il simbolismo della cifra dieci nell'Apocalisse di San Giovanni
}

\section{Symbolism of the number ten in the Apokalypse of John}

\section{Symbolika liczby dziesięć w Apokalipsie św. Jana}

Parole chiavi: Apocalisse di san Giovanni, simbolismo artmetico, numero dieci, drago, bestia, corni, diademi.

Key words: St. John's Apocalypse, symbolism of numbers, number ten, evil, dragon, beast, horns, diadems.

Słowa klucze: Apokalipsa św. Jana, symbolizm artmetyczny, liczba dziesięć, smok, bestia, rogi, diademy.

$\mathrm{L}$ eggendo l'ultimo libro del Nuovo Testamento il lettore si accorge subito che "il simbolismo occupa, nell'interpretazione dell'Apocalisse, un posto centrale. [...] e del resto è un fatto che si impone a una prima lettura: per comprendere l'Apocalisse occorre interpretare i suoi simboli" 1 . In tal modo "l'autore del libro presuppone e richiede un soggetto interpretante [...] e il lettore o ascoltatore viene coinvolto nel dramma raccontato come un ermeneuta sapiente" ${ }^{2}$. Seguendo la proposta di U. Vanni possiamo distinguere le seguenti costanti simboliche dell'Apocalisse: il simbolismo $\operatorname{cosmico}^{3}$,

1 U. Vanni, L’Apocalisse. Ermeneutica, esegesi, teologia, Bologna 1991, p. 31.

2 G. Segalla, Apocalisse di Giovanni. In un mondo ingiusto la visione di un mondo giusto, Reggio Emilia 2004, p. 13.

3 Cf. U. Vanni, L’Apocalisse, p. 34-35; S. Gądecki, Wstęp do pism Janowych, Gniezno ${ }^{2}$ 1996, pp. 158-159; M. Karczewski, Reinterpretacja Księgi Rodzaju w Apokalipsie św. Jana, Olsztyn 2010, p. 60. 
gli sconvolgimenti cosmici ${ }^{4}$, il simbolismo teriomorfico ${ }^{5}$, il simbolismo antropologico $^{6}$, il simbolismo cromatico $^{7}$ e il simbolismo aritmetico ${ }^{8}$. Naturalmente il simbolismo della cifra dieci che ci interessa particolarmente fa parte del simbolismo aritmetico che trova "uno spazio relativamente ampio" nell'Apocalisse. Inizieremo quindi la nostra riflessione con uno sguardo sommario sulla problematica di questa costante simbolica nell'Apocalisse. In seguito daremo qualche osservazione sullo sviluppo del simbolismo della cifra in genere e nella Bibbia per trovare eventuali punti di contatto fra quest'uso della cifra e il testo apocalittico. La terza parte conterrà l'esegesi dei singoli passi dell'Apocalisse in cui ricorre il numero dieci. Con un certo numero di commentatori rappresentativi dell'Apocalisse, faremo il punto sullo stato attuale della esegesi di ogni testo. Per dirla in sintesi, con le pagine che seguono intendiamo gettare uno sguardo esplorativo e allo stesso tempo sistematico sul significato simbolico del numero dieci nel libro dell'Apocalisse.

4 Cf. U. Vanni, L’Apocalisse, pp. 35-37.

5 Cf. U. Vanni, L’Apocalisse, pp. 38-40; W. Michniewicz, Symbolika chromatyczna i zoomorficzna w Apokalipsie św. Jana, in: K. Korotkich, J. Ławski (ed.), Apokalipsa. Symbolika Tradycja - Egzegeza 1, Białystok 2006, pp. 64-71.

6 Cf. U. Vanni, L’Apocalisse, p. 40-49; L. Pedroli, Dal fidanzamento alla nuzialità escatologica. La dimensione antropologia del rapporto tra Cristo e la Chiesa nell'Apocalisse, Assisi 2007, pp. 39-119.

7 Cf. U. Vanni, L’Apocalisse, pp. 49-52; W. Michniewicz, Symbolika, pp. 60-64.

8 Cf. U. Vanni, L'Apocalisse, p. 52-55; A. Yarbro Collins, Numerical Symbolism in Jewish and Early Christian Apocalyptic Literature, in: W. von Haase (ed.), Aufstieg und Niedergang der Römischen Welt, Berlin-New York 1984, pp. 1221-1287; G. Biguzzi, I numeri nell'Apocalisse di Giovanni e il loro linguaggio, LA 50(2000), pp. 143-166; L. Díez Merino, La numerología en el Apocalipsis: un principio de hermenéutica, CTom 127(2000), pp. 59-98; N. Marconi, Le mille immagini dell'Apocalisse. Una introduzione al linguaggio audiovisivo dell'Apocalisse, Milano 2002, pp. 64-75; E. Corsini, I numeri nell'Apocalisse, in: E. Bosetti, A. Colacrai (ed.), Apokalypsis. Percorsi nell'Apocalisse in onore di Ugo Vanni, Assisi 2005, pp. 391-416; B. Zbroja, Symbolika arytmetyczna Apokalipsy, in: T. Jelonek (ed.), Inkulturacja Biblii, Kraków 2007, pp. 131-158.

9 U. Vanni, L'Apocalisse, p. 52. A. Yarbro Collins, Numerical Symbolism, pp. 1272.1286 afferma che l'ultimo libro del Nuovo Testamento detiene il primato, non solo all'interno del Nuovo Testamento, ma anche nei confronti di tutta la letterattura apocalittica. Considerando tutti i numeri dell'Apocalisse (cardinali, ordinali, frazionali) sono 275, insieme invece con quelli che entrano in composizione sono 283. Cf. G. Biguzzi, I numeri nell'Apocalisse, p. 143, n. 2 . 


\section{Il simbolismo aritmetico nell'Apocalisse}

Quando prendiamo in esame il fenomeno del simbolismo aritmetico facilmente ci rendiamo conto che questa costante simbolica consiste nello spostamento di significato che "può essere identificato nel fatto che la quantità, di per sé neutra, espressa dai numeri, assume mediante scelte artificiose o alterazioni, un valore che è qualitativo"10. Troviamo, allora, i numeri, privati completamente del valore quantitativo, che assumono un altro valore del tutto diverso. Come esempio assai significativo si può citare la cosiddetta gematria che „consisteva nell'esprimere ogni lettera di una parola con il valore numerico ad essa corrispondente, fare la somma, e poi parlare soltanto nella forma velata del numero" 11 . Poi abbiamo anche le iperboli numeriche che vogliono suggerire l'idea di una dimensione al di là dell'immaginato ${ }^{12}$.

Tra i tanti numeri usati simbolicamente dall'autore dell'Apocalisse troviamo anzitutto il numero sette, che viene menzionato 55 volte su un totale di 88 volte nel NT. Questa cifra già nell'ambiente veterotestamentario, e ancora prima, indica la completezza e la totalità ${ }^{13}$. Come afferma U. Vanni: "è un dato che l'autore dell'Apocalisse accoglie dal suo ambiente culturale e considera acquisito. L'impronta qualitativa di significato non deriva da lui" ${ }^{14}$. La creatività

10 U. Vanni, L’Apocalisse, p. 52.

11 E. Lohse, L'Apocalisse di Giovanni, Brescia 1974, p. 142. L’esempio più esplicito della gematria che troviamo nell'Apocalisse è il 666 di 13,18. Anche se le intepretazioni proposte sono varie, quella più diffusa propone leggere il numero 666 come l'equivalente di Nerone Cesare che si ottiene sommando insieme i valori numerici delle lettere ebraiche che lo esprimono (NRWN QSR: $\mathrm{n}=50+\mathrm{r}=200+\mathrm{w}=6+\mathrm{n}=50+\mathrm{q}=100+\mathrm{s}=60+\mathrm{r}=200$ : totale 666), cf. O. Rühle, $\alpha \rho \iota \theta \mu \epsilon ́ \omega, \alpha \rho \iota \theta \mu o ́ \varsigma$, in: Kittel G., Friedrich G. (ed.), Grande Lessico del Nuovo Testamento, [citato in seguito come GLNT], vol. I, Brescia 1965, col. 1235. Notiamo che non tutti sono d'accordo con questa interpretazione, cf. E. Corsini, Apocalisse prima e dopo, Torino 1980, pp. 342-346. La rassegna delle interpretazioni antiche e moderne si trova nell'articolo di G. Biguzzi, I numeri nell'Apocalisse, pp. 156-163. L'esegeta italiano parla anche dei tentativi di interpretare il 666 in base al simbolismo del 6 e di mettere il 666 in relazione al 12 e non al 7. Secondo F. Kawka, Odkrycie i złamanie szyfru Biblii. Tajemnice języka liczb Jezusa w Ewangelii i Apokalipsie św. Jana, Katowice 2000, pp. 27-54 il numero 666 si riferisce simbolicamente al profeta Elia.

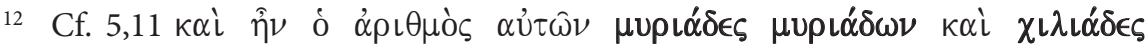

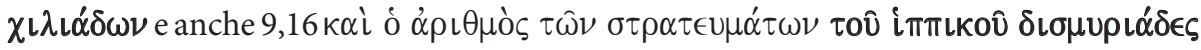

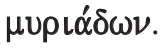

13 K.H. Rengstorf, é $\pi \tau \dot{\alpha}$, in: GLNT, vol. III, Brescia 1967, col. 807-813.

14 U. Vanni, L’Apocalisse, p. 53. 
dell'autore si rivela sopratutto nell'applicazione che ne fa sia a livello esplicito ${ }^{15}$, sia a livello di strutturazione letteraria del libro $^{16}$, nelle enumerazioni di sette elementi. In tutti e due i casi, l'autore ci vuole indicare sempre un tipo di totalità che poi il contesto determina e chiarisce ${ }^{17}$.

I numeri usati più frequentemente dall'autore con il loro significato simbolico sono ${ }^{18}$ : due per rendere valida la testimonianza dei due testimoni $(11,1-13)$, poi la bestia ha anche "le due corna" $(13,11)$ che diventa "un rimando ironico, un contraltare della testimonianza veritiera dei due martiri del cap. $11^{\prime \prime 19}$; il numero tre che designa il divino ${ }^{20}(1,4.5 .8 ; 4,8.9 .11 .18 ; 12,10)$ e anche riguarda il demoniaco che tenta di imitare Dio $(16,13 ; 17,8)$; tre e mezzo (la metà di sette) che esprime "una totalità dimezzata, una parzialità"21 $(11,2$ [42 mesi $=3$ anni e mezzo].3; 12,6 [similmente 1260 giorni $=3$ anni e mezzo]); quattro che è il numero della terra con i suoi angoli e i quattro venti $(7,1)^{22}$; il numero cinque che esprime una limitezza $(9,5.10)$; il numero dodici "che non ha riscontri persuasivi nella letteratura apocalittica e sembra

15 L'autore enumera: 9 v. sette angeli $(8,2.6 ; 15,1.6 .7 .8 ; 16,1 ; 17,1 ; 21,9) ; 5$ v. sette stelle $(1,16.20 \mathrm{bis} ; 2,1 ; 3,1) ; 5 \mathrm{v}$. sette teste $(12,3 ; 13,1 ; 17,3.7 .9) ; 4$ volte sette chiese $(1,4.11 .20 \mathrm{bis})$; 4 v. sette spiriti $(1,4 ; 3,1 ; 4,5 ; 5,6) ; 4$ v. sette candelabri $(1,12.20$ bis; 2,1$) ; 4$ v. sette flagelli $(15,1.6 .8 ; 21,9) ; 4 \mathrm{v}$. sette coppe $(15,7 ; 16,1 ; 17,1 ; 21,9) ; 3 \mathrm{v}$. sette sigilli $(5,1.5 ; 6,1) ; 3 \mathrm{v}$. sette tuoni $(10,3.4 \mathrm{bis}) ; 2 \mathrm{v}$. sette trombe $(8,2.6) ; 2 \mathrm{v}$. sette re $(17,9.11) ; 1 \mathrm{v}$. sette lampade $(4,5) ; 1 \mathrm{v}$. sette corna $(5,6)$; $1 \mathrm{v}$. sette occhi $(5,6)$; $1 \mathrm{v}$. settemila $(11,13)$; $1 \mathrm{v}$. sette diademi $(12,3), 1 \mathrm{v}$. sette colli $(17,9)$.

16 Nella struttura del libro abbiamo i settenari: delle lettere, dei sigilli, degli angeli con le trombe, degli angeli con le coppe. Si tratta sempre della successione concatenata di sette elementi. Per la spiegazione cf. U. Vanni, La struttura letteraria dell'Apocalisse, Brescia 1980, pp. 120-130 e E. Corsini, Apocalisse, pp. 84-89.

17 Cf. U. Vanni, L'Apocalisse, p. 53.

18 Lanalisi dettagliata di tutti i numeri presenti nell'Apocalisse si trova nell'articolo di B. Zbroja, Symbolika arytmetyczna Apokalipsy, pp. 131-158.

19 N. Marconi, Le mille immagini dell'Apocalisse, p. 65, cf. anche E. Corsini, I numeri nell'Apocalisse, pp. 407-411.

20 Cf. D. Piekarz, Orędzie na nasze czasy. Księga Apokalipsy, Zeszyty Formacji Duchowej 53(2011), p. 18.

21 U. Vanni, L'Apocalisse, p. 53. Secondo l'autore la stessa idea di una totalità diven-

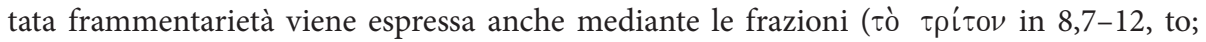

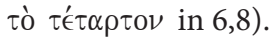

22 Secondo N. Marconi, Le mille immagini dell'Apocalisse, p. 68 „non deve stupire che le formule, per quanto variate, che fanno riferimento ai popoli della terra siano quaternarie $(5,9 ; 9,9 ; 10,11 ; 11,9 ; 13,7)$ e che quando è tutta la terra che loda Dio, questa lode sia quaternaria e non ternaria $(5,13)$, così come l'azione divina diventa quatenaria se si rivolge a tutta la terra $(8,5 ; 9,15 ; 16,18)$ ". 
una derivazione diretta dell'autore dalle 12 tribù di Israele e dai 12 apostoli" 23 $(7,5-8 ; 12,1 ; 21,12-21)$; il numero mille esprime invece "la totalità propria del livello di Dio e dell'azione di Cristo"24 (20,1-6).

Quello che è tipico per l'Apocalisse non è solo l'uso simbolico dei singoli numeri, ma anche la loro combinazione mediante operazioni aritmetiche. Come esempio assai interessante possiamo citare la cifra 144.000 risultante dalla moltiplicazione $12 \times 12 \times 1000$. Secondo la spiegazione di U. Vanni "si avrebbe una moltiplicazione ideale tra le 12 tribù di Israele e i 12 apostoli dell'agnello: Antico e Nuovo Testamento si compenetrerebbero così al punto da formare un unico popolo di Dio, ma che risulta maggiorando in una unità superiore e dinamica i valori presenti nel periodo dell'Antico e del Nuovo Testamento. La successiva moltiplicazione per 1000 rapporta questo popolo di Dio [...] ai 1000 anni propri della presenza attiva di Dio e di Cristo nella storia delluomo"25.

\section{La cifra dieci nella Bibbia al di fuori dell'Apocalisse}

Come afferma F. Hauck alla base "dell'importanza attribuita al numero dieci sia in Israele che in altri popoli sta l'abitudine originaria di numerare sulle dita di una o di entrambe le mani, grazie alla quale il numero dieci diviene la base del sistema decimale, in uso nell'antichità accanto al sistema duodecimale"26.

Il numero dieci nell'Antico Testamento esprime una quantità approssimativa che può essere superiore al dieci stesso o anche inferiore. Come esempio citiamo Nm 14,22 dove Dio parlando degli ebrei ribelli dice che essi l'hanno tentato dieci volte senza obbedire alla sua voce. In questo caso il numero dieci significa: più volte ${ }^{27}$. La cifra dieci ricorre nel Primo Testamento in alcune serie di notevole importanza: Dio concentra ed esprime la sua divina volontà in dieci precetti (Es 20; Dt 5), le dieci piaghe manifestano progressivamente la forza di Dio in Egitto (Es 7-11), l'epoca che precede il diluvio comprende

23 U. Vanni, L'Apocalisse, p. 54. Similmente la cifra ventiquattro che si riferisce ai vegliardi, interpretati spesso come l'immagine della Chiesa dei due Testamenti: i dodici patriarchi, più dodici apostoli $(4,4)$ oppure come l'immagine della Chiesa vista come l'unico vero Israele che si sviluppa sul fondamento delle dodici tribù, cf. N. Marconi, Le mille immagini dell'Apocalisse, pp. 74-75. Sul simbolismo del numero dodici e dei suoi multipli, cf. E. Corsini, I numeri nell'Apocalisse, pp. 404-407.

24

25

26

27

U. Vanni, L'Apocalisse, p. 53.

U. Vanni, L'Apocalisse, p. 54.

F. Hauck, $\delta \epsilon ́ \kappa \alpha$, in: GLNT, vol. II, Brescia 1966, col. 829.

Cf. P. Prigent, L'Apocalisse di S. Giovanni, Roma 1985, p. 92. 
dieci patriarchi (Gen 5), il tributo sacro che deve essere pagato a Dio è la decima (Gen 28,22). In questi casi il numero dieci potrebbe avere un valore totalizzante ed esprimere l'idea di una completezza e totalità ${ }^{28}$.

Il numero dieci compare anche nelle misure dell'arca (Gen 6,15), del tabernacolo nel deserto (Es 26,27) e del tempio (1 Re 6,7) ${ }^{29}$. Può indicare anche un indeterminato periodo del tempo, ma relativamente breve: al termine di dieci anni da quando Abram abitava nel paese di Canaan, Sarai prende la schiava egiziana Agar e la dà al marito (Gen 16,3); ogni dieci giorni veniva offerto vino per tutti in abbondanza sotto l'amministrazione di Neemia ( Ne 8,18); al termine di dieci giorni la parola del Signore fu rivolta a Geremia (Ger 42,7), la prova di Daniele, Anania, Misaele e Azaria durava per dieci giorni (Dn $1,12-15)^{30}$.

Nel Nuovo Testamento la nostra cifra ricorre 24 volte $^{31}$. Essa viene usata spesso come numero tondo per i valori bassi (le dieci vergini in Mt 25,1; i dieci talenti in Mt 25,28; i dieci lebbrosi in Lc 17,11-19; le dieci mine in Lc 19,1126). Due volte la cifra dieci viene usata per chiamare il resto dei discepoli (Mt 20,24; Mc 10,41) $)^{32}$. Ed una volta il numero dieci serve per determinare

28 Cf. H.A. Brongers, Die Zehnzahl in der Bible und in ihrer Umwelt, in: W.C. van Unnik, A.S. van der Woude (ed.), Studia biblica et semitica Theodoro Christiano Vriezen qui munere professoris theologiae per XXV annos functus est, ab amicis, collegis, discipulis dedicata, Wageningen 1966, pp. 30-45.

29 Cf. F. Hauck, $\delta$ é $\alpha \alpha$, col. 830-832. L'autore parla anche del significato della cifra dieci nel giudaismo (Abramo giunge a perfezione attraverso dieci tentazioni ed è con dieci parole di Dio che è stato creato il mondo; importante è il numero dieci nel culto, poiché vi è una regola che stabilisce che con la presenza di dieci adulti si può avere un vero atto cultuale, nel quale si rende presente la Shekinà), nell'apocalittica e in Filone, per il quale il numero dieci è il numero della perfezione.

30 Cf. D.E. Aune, Revelation 1-5, Nashville 1997, p. 166.

31 Mt 20,24; 25,1.28; Mc 10,41; Lc 14,31; 15,8; 17,12.17; 19,13bis.16.17.24.25; At 25,6; Ap 2,10; 12,3; 13,1bis; 17,3.7.16; 17,12bis. F. Hauck, $\delta \epsilon ́ \kappa \alpha$, col. 832-833 nota anche che nel Nuovo Testamento abbiamo esempi dei dieci elementi elencati: in Mt 8-9 è la somma dei miracoli che mostrano il potere messianico di Gaesù; talvolta la cifra dieci inquadra delle enumerazioni, come in $\mathrm{Rm} 8,38$-39 (le dieci potenze che non sono in grado di separare i fedeli da Dio), e in 1 Cor 6,9-10 (i dieci vizi che escludono dal regno di Dio).

32 Normalmente si usa il numero dodici per chiamare il collegio dei discepoli. Due testi citati sopra parlano della situazione in cui la madre dei figli di Zebedèo parla con Gesù e chiede per i suoi figli il posto alla destra e alla sinistra del Signore. L'evangelista raccontando la reazione degli altri apostoli li chiama con il numero dieci per far capire che sono tutti gli Apostoli tranne i due figli di Zebedeo. 
il tempo di dieci giorni (At 25,6) e descrive un breve periodo del soggiorno di Festo a Gerusalemme.

\section{L'analisi esegetico-simbolica delle ricorrenze del numero dieci nell'Apocalisse}

Veniamo adesso al testo dell'Apocalisse. Come abbiamo già accennato il numero dieci $\left(\delta \delta^{\prime} \kappa \alpha\right)$ si trova 9 volte $(2,10 ; 12,3 ; 13,1 \text { bis; 17,3.7.12bis.16) })^{33}$. Intendiamo analizzare le ricorrenze della cifra dieci nel testo del libro per capire come l'autore dell'Apocalisse usi questo numero e se possiamo parlare di un certo sviluppo della sua simbolica.

\subsection{Una tribolazione di dieci giorni $(2,10)$}

Per la prima volta il numero dieci ricorre nella lettera alla chiesa di Smirne $(2,8-$ 11). Lantica città commerciale greca di Smirne era conosciuta nell'antichità per le sue ricchezza e la prosperità economica ${ }^{34}$, perché "al tempo dell'Apocalisse era centro di intensi scambi commerciali tra mare ed entroterra anatolica, da cui emergevano carovane di cammelli con carichi di merci e manufatti orientali" 35 .

La lettera indirizzata alla comunità cristiana della città inizia con lautopresentazione del Cristo $(2,8)$ che richiama la prima coppia di titoli usati nella cristofania (1,9-20): "il Primo e l'Ultimo" in 1,17 e "il Vivente, e fui morto" in 1,18 . Questi termini si riferiscono al dominio del tempo e alla vittoria pasquale della vita sulla morte ${ }^{36}$.

La vita dei critiani smirioniti è segnata dalla tribolazione e della povertà $(2,9)$, che in realtà viene riconosciuta dal Cristo come una vera richezza, quella

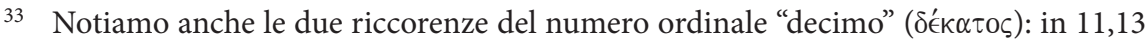
si parla di un grande terremoto che distrugge un decimo della città, inveve in 21,20 viene menzionato il decimo fondamento di Gerusalemme che è la pietra di crisopraso (o olivina). La rovina di un decimo della città può significare la mitezza del castigo (appena un decimo della città), cf. P. Ostański, Objawienie Jezusa Chrystusa. Praktyczny komentarz do Apokalipsy, Ząbki 2005, p. 210. Secondo B. Zbroja, Symbolika arytmetyczna Apokalipsy, p. 148 il numero decimo si riferisce qui alla possibille conversione dell'uomo, perchè dopo la rovina di un decimo i sopravvissuti diedero gloria al Dio del cielo.

34 Cf. E. Lohse, LApocalisse, p. 54.

35 G. Biguzzi, Apocalisse, Milano 2005, p. 109.

36 Cf. P. Podeszwa, Paschalna pamięć o Jezusie. Studium egzegetyczno-teologiczne wyra-

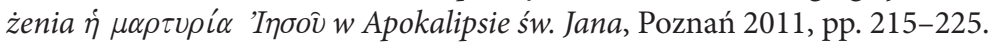


della fede. I cristiani soffrono la persecuzione e le bestemmie "di coloro che dicono di essere giudei e non lo sono, ma (sono) una sinagoga di Satana" $(2,9)$. Probabilmente si tratta della locale sinagoga che cercava di opporsi allespansionismo cristiano ${ }^{37}$. Il conflitto sembra molto forte e i seguaci di Gesù dovranno affrontare ancora altre sofferenze: alcuni dei suoi membri saranno gettati in carcere. Questa persecuzione, messa in atto dagli uomini,

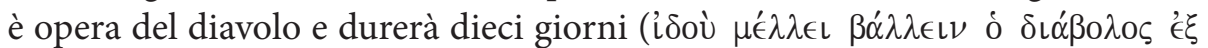

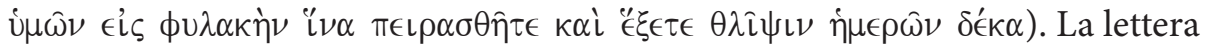
annuncia quindi una "tribolazione di dieci giorni" che sarà una dura prova per i cristiani di Smirna. Secondo molto autori il numero dieci indica qui un tempo breve delle persecuzioni ${ }^{38}$. G. Biguzzi afferma il contrario: "poiché per esperienza primordiale ogni persona collega il numero «10» con il numero della dita delle mani, una tribolazione di dieci giorni è una tribolazione lunga, essendo necessarie tutte e dieci le dita delle mani per fare il conteggio" ${ }^{39}$. Se la cifra dieci ha anche "un valore totalizzante, dovremmo dedurne che il periodo indicato, breve o lungo che sia, rappresenti la totalità della persecuzione" 40 . Il numero dieci diventa qui il numero dell'agire di Satana (ó $\delta\llcorner\alpha ́ \beta \beta$ della forza contraria a $\mathrm{Dio}^{42}$, ma Egli rimane padrone della situazione e mette i suoi alla prova, però soltanto per un determinato tempo. Sarebbe dunque un periodo sufficientemente lungo, ma limitato ${ }^{43}$. Il numero dieci avrebbe qui il significato simbolico di esprimere l'idea di un tempo che sembra abbastanza lungo e senza fine, ma in realtà è un tempo limitato. Sarebbe l'affermazione del carattere limitato della persecuzione, per volere di Dio. Dieci sarebbe dunque

37 Così G. Biguzzi, Apocalisse, p. 110. Secondo l'autore ci sono due possibilità: i giudei menzionati in 2,9 potrebbero essere la porzione giudeo-cristaina della comunità di Smirne in rotta di collisione con la parte etnico-cristiana, oppure la locale sinagoga. Secondo Biguzzi la seconda ipotesi è più probabile. Cf. anche D. Kotecki, Duch Święty w zgromadzeniu liturgicznym w świetle Apokalipsy św. Jana, Warszawa 2006, pp. 94-99.

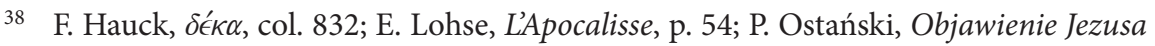
Chrystusa, p. 97; B. Zbroja, Symbolika arytmetyczna Apokalipsy, p. 147; F. Sieg, Apokalipsa św. Jana. Listy do siedmiu Kościołów (Ap 1-3), Pelplin ${ }^{2} 2009$, p. 83.

39 G. Biguzzi, Apocalisse, p. 111.

40 E. Lupieri, L’Apocalisse di Giovanni, [Roma] ${ }^{4} 2005$, p. 126.

41 Cf. G. Biguzzi, I numeri nell'Apocalisse, p. 147.

42 Cf. D. Piekarz, Orędzie na nasze czasy, p. 19.

43 Cf. E. Bianchi, L'Apocalisse di Giovanni. Commento esegetico-spirituale. Magnano 1988, p. 61; G. Ravasi, Apokalipsa, Kielce 2002, p. 30. 
un numero tondo, appropriato allo scopo che sembra indicare "una limitatezza nonostante l'apparenza del contrario" 44 .

\subsection{Un Drago con dieci corna $(12,3)$}

Siamo al capitolo 12 che contiene la famosa visione della Donna avvolta

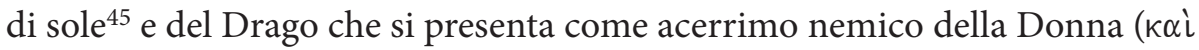

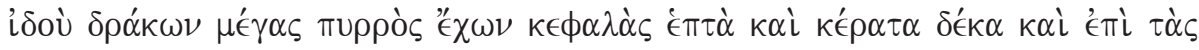

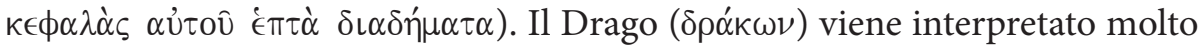
spesso come il Satana - serpente di Gen 3 che si contrappone alla Donna e in prospettiva alla sua discendenza $(\text { Gen } 3,15)^{46}$. Il suo aspetto spaventoso viene descritto con tratti tradizionali (cf. Dan 7,7): "grande" ( $\mu$ '́ $\gamma \alpha \varsigma$ ), cioè

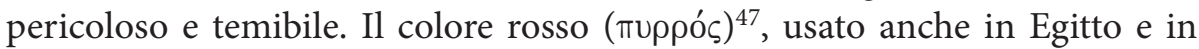
Babilonia per dipingere i mostri tenebrosi, ne indica i propositi omicidi ${ }^{48}$. Il colore rosso potrebbe anche essere un'allusione simbolica al sangue di Cristo oppure al potere di Roma ${ }^{49}$. Il Drago ha sette teste (' $\left.\epsilon \omega \nu \quad \kappa \in \phi \alpha \lambda \grave{\alpha} \varsigma \dot{\epsilon} \pi \tau \dot{\alpha}\right)$. Seguendo il simbolismo aritmetico del numero sette l'autore esprime l'idea che il drago ha una totalità di vitalità ${ }^{5}$, è attivo e ha potere. Così il Drago diventa la massima espressione del male ${ }^{51}$. Sulle sue sette teste ha dieci corna (к'́́ $\left.\rho \alpha \tau \alpha \delta^{\prime} \in \alpha\right)$.

Veniamo direttamente al simbolismo delle dieci corna. Il corno è il simbolo teriomorfo per indicare la forza, già diffusissimo nell'Antico Testamento, dove il corno è simbolo di potenza (Nm 23,22; Dt 33,17; 1 Sam 2,1; 1 Re 22,11, Sal 18,3 ) e di dignità regale (Sal 112,9; 148,14; Zc 2,1; Dn 7,7.20; 8,3). Nel Primo Testamento - a differenza dell'ambiente greco - il corno non soltanto esprime la potenza fisica nella simbologia profetica, ma è l'espressione diretta della

\footnotetext{
44 U. Vanni, L’Apocalisse, p. 54.

45 Cf. P. Farkaš, La „Donna” di Apocalisse 12. Storia, bilancio, nuove prospettive, Roma 1997.

46 Sull'interpretazione del Drago, cf. D. Kotecki, Duch Święty w zgromadzeniu liturgicznym, pp. 57-70.

47 Sul significato del colore $\pi u \rho \rho o ́ \varsigma$ cf. M. Karczewski, Reinterpretacja Księgi Rodzaju, pp. $64-65$, n. 82 .

48 Cf. E. Lohse, L’Apocalisse, p. 125.

49 Cf. P. Farkaš, La „Donna” di Apocalisse 12, p. 217.

50 D. H. Lawrance, Apocalisse, Roma 1995, p. 77 scrive "sette teste, sette vite".

51 Cf. U. Vanni, L’Apocalisse, pp. 242-243.
} 
potenza $^{52}$. Il testo anticotestamentario che potrebbe essere la fonte di questa immagine è il libro di Daniele, particolarmente la quarta Bestia che sorge dal mare $(7,7)$ e ha dieci corna che forse sono i successori di Alessandro Magno, precisamente i Seleucidi di Siria ${ }^{53}$. Le corna sono quindi un simbolo del potere regale (in Ap 17,12 si dice espressamente che le dieci corna della Bestia sono i dieci re).

Abbiamo già detto che il Drago è la massima espressione del male, ma il male, in tutta la pienezza della sua vitalità ( $\kappa € \phi \alpha \lambda \grave{\alpha} \varsigma \dot{\epsilon} \pi \tau \dot{\alpha})$, è sempre limitato: il numero dieci delle corna indica una potenza limitata nonostante la sua grandezza che potrebbe sembrare smisurata a livello terrestre ${ }^{54}$. Attraverso il simbolismo aritmetico della cifra dieci l'autore esprime un'idea di una forza limitata nonostante l'apparenza minacciosa di potenza travolgente, suggerita dal simbolismo teriomorfo usato. Similmente a 2,10 il numero dieci si riferisce alla potenza opposta a Dio.

Notiamo subito anche un altro particolare molto interessante. Il numero dieci delle corna supera il numero sette delle teste (similmente in 13,1). Forse "la sproporzione tra il 10 e il 7 è intenzionale e parla, da un lato di arrogante ostenzione di potenza, e, dall'altra, di disordine e di caos" ${ }^{\prime 25}$.

L'immagine simbolica del Drago viene completata ancora dall'indicazione

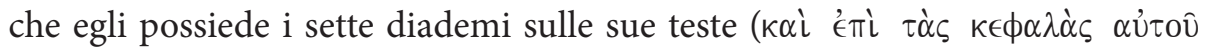
$\dot{\epsilon} \pi \tau \dot{\alpha} \delta\left\llcorner\alpha \delta \eta_{\mu} \mu \tau \tau \alpha\right)^{56}$. Il diadema, l'insegna tipica del re, simboleggia un potere regale, umano, politico, terrestre. In questo modo l'autore esprime l'idea che la pienezza della vitalità del Drago si concretizza tutta nella storia umana in contatto coi suoi centri di potere ${ }^{57}$.

52 Cf. W. Foerster, אé $\rho \alpha \varsigma$, in: GLNT, vol. V, Brescia 1967, col. 351.

53 Cf. E. Corsini, I numeri nell'Apocalisse, p. 399; M. Parchem, Księga Daniela, Częstochowa 2008, pp. 455-456.

54 Cf. H. Gollinger, Das Grosse Zeichen von Apokalypse 12, Stuttgart 1971, p. 87.

55 G. Biguzzi, I numeri nell'Apocalisse, p. 153.

56 Secondo E. Lohse, L'Apocalisse, p. 126 l'informazione che su ogni testa si trova un diadema probabilmente accenna già al rapporto tra il Drago e la Bestia che apparirà adornata di diademi in 13,1 .

57 Cf. U. Vanni, L’Apocalisse, p. 243. 


\subsection{Una Bestia che aveva dieci corna e sulle corna dieci diademi (13,1bis)}

Quando il Drago si avvicina alla riva del mare $(12,18)$ e appena si ferma sulla spiaggia, il Veggente vede una nuova immagine. Dal mare si erge una Bestia ${ }^{58}$ :

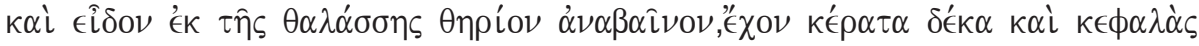

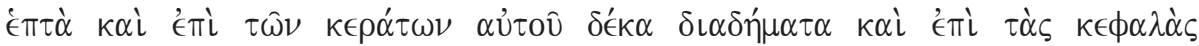

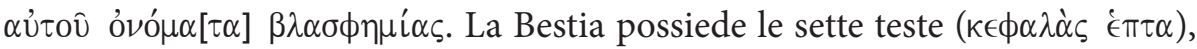

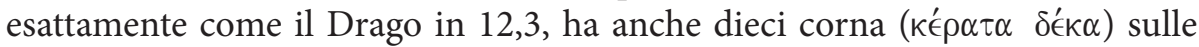

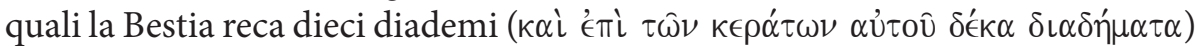
come segno del suo potere regale, del quale vuole servirsi nella sua qualità di antagonista di Cristo. I primi due elementi della descrizione della Bestia permettono di vedere la grande somiglianza tra il Drago e la Bestia: hanno le sette teste che simboleggiano la grande vitalità e le dieci corna che, come abbiamo già detto, esprimono una potentissima forza, ma limitata nella sua misura. Ma la grande somiglianza non significa l'identità: dapprima notiamo che nella descrizione della Bestia l'ordine degli elementi è diverso (qui prima i corni e poi le teste), e poi, secondo 12,3 il Drago ha sulle sue teste i sette diademi, invece in 13,1 i diademi sono dieci e vengono distribuiti sulle dieci corna della Bestia. Il termine diadema ricorre nell'Apocalisse solo in 12,3; 13,1; 19,12 e sottolinea lo svolgimento in atto di un potere regale, che può essere positivo $(19,12)$ o negativo $(12,3 ; 13,1)$. Il diadema è allora il simbolo tipico dei re ed evoca l'idea di regalità. Adesso i diademi sono dieci legati alle dieci corna: la potenza travolgente della Bestia (dieci corna) si concretizza tutta, similmente al potere del Drago, nella storia umana ancora di più attraverso i grandi centri del potere politico ed economico sulla terra (dieci diademi). Il passaggio dei diademi dalle teste alle corna potrebbe esprimere sul piano simbolico l'uguaglianza in dignità tra i sovrani umani e quelli demoniaci. Il rapporto tra la Bestia e i re della terra è maggiormente sottolineato dal fatto che sulle teste

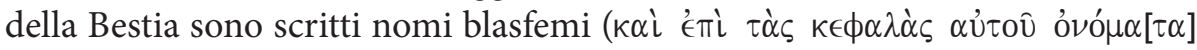
$\left.\beta \lambda \alpha \sigma \phi \eta \mu i \alpha_{\varsigma}\right)$ che offendono l'onore di Dio ${ }^{59}$. Il Drago diede alla Bestia la sua potenza, il suo trono e una potestà grande $(13,2)$. Questa investitura mette

58 Sull'interpretazione della Bestia dal mare, cf. D. Kotecki, Duch Święty w zgromadzeniu liturgicznym, pp. 70-81.

59 Ci sono varie interpretazioni. Se accettiamo ó $\nu$ ó $\alpha \alpha[\tau \alpha]$ al plurale, la variante bene attestata e contenuta in buoni manoscritti (l'Alessandrino e il maiuscolo 046) si tratta di titoli attribuiti a sovrani nel quadro del culto imperiale romano: eccelso, divino, figlio di dio, signore e dio, salvatore ecc. Quando un uomo si fregia di questi titoli, Dio è bestemmiato; cf. E. Lohse, L’Apocalisse, p. 135. Se si sceglie la lezione "nome blasfemo", si pensa prima di tutto 
la Bestia in primo piano ed essa condurrà la guerra contro i santi $(13,7)$ che il Drago aveva intrapreso contro la discendenza della Donna $(12,17)^{60}$.

La Bestia in 13,1 possiede quindi le dieci corna e sulle dieci sue corna i dieci diademi. Secondo il simbolismo delle corna, dei diademi e soprattutto del numero dieci si può interpretare quest'immagine come l'espressione di una forza e di una regalità limitata, nonostante l'apparenza minacciosa di potenza travolgente, suggerita dal simbolismo teriomorfo della Bestia. Questa potenza (corna) della Bestia si incarna nella struttura della storia umana (diademi), ma è sempre la realtà considerata nella sua limitatezza (dieci). La Bestia sarà sconfitta dalla vittoria dell'Agnello che ha sette corna (la pienezza della forza in 5,6$)^{61}$ e dal Cavaliere bianco del capitolo 19 che cinge innumerevoli diademi $(19,20)^{62}$.

\subsection{Una Bestia con sette teste e dieci corna $(17,3.7)$}

Nel capitolo 17 inizia il racconto sul giudizio di Babilonia $(17,1-19,10)$. In 17,3 il Veggente, condotto in spirito nel deserto, vede una Bestia. Questa volta viena descritta come una Bestia scarlatta che è piena di nomi blasfemi. Essa ha

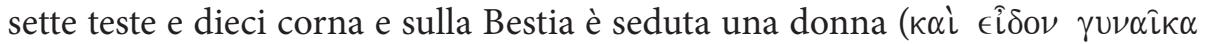

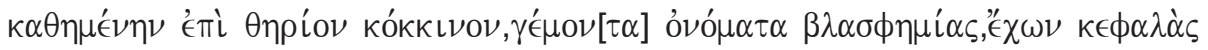
$\left.\dot{\epsilon} \pi \tau \dot{\alpha} \kappa \alpha \grave{\iota} \kappa \dot{\epsilon} \rho \alpha \tau \alpha \delta^{\prime} \in \kappa \alpha\right)$. La presenza delle sette teste e delle dieci corna permette di identificarla con la Bestia che abbiamo già incontrato al capitolo 13,1, la cui sovranità si estende a tutta l'ecumene. Come anche in 13,1 la Bestia ha una vitalità ed una potenza grandissime (sette teste), ma la sua forza (corna) rimane limitata (dieci).

In seguito la visione si concentra sulla donna seduta sulla Bestia (17,46). La donna possiede un nome scritto, misterioso: "Babilonia la grande, la madre delle meretrici e degli abomini della terra". Essa è ebbra del sangue dei santi e del sangue dei testimoni di Gesù $(17,6)^{63}$. Il Veggente rimane preso da grande meraviglia $(17,6)$ e gli viene data l'interpretazione dell'immagine che lui ha contemplato. Langelo sta per spiegare il senso della visione, svelandone il mistero. Con le parole del 17,7 l'angelo preannuncia la spiegazione che lui

al titolo più diffuso, quello di Augusto, che significa l'adorabile, accuratamente inteso in senso forte (adorato); cf. P. Prigent, L'Apocalisse, p. 402.

60 Cf. G. Biguzzi, Apocalisse, p. 254.

61 Cf. P. Podeszwa, Paschalna pamięć o Jezusie, pp. 230-245.

62 Cf. ibidem, p. 290.

63 Sull'intepretazione della donna prostituta, cf. D. Kotecki, Duch Święty w zgromadzeniu liturgicznym, pp. 81-94. 


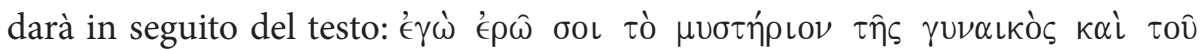

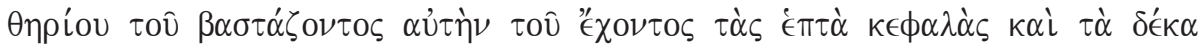
$\kappa ' \in ́ \rho \tau \alpha$. Poi segue la spiegazione $(17,8-18)$, anche se il mistero rimane sempre e ci vuole "l'intelletto che ha saggezza" $(17,9)$. Notiamo che l'ordine della spegazione è un po' diverso da quello preannunciato prima in 17,7 (donna, Bestia, sette teste, dieci corna). Si tratta successivamente della Bestia (v. 8) ${ }^{64}$, delle teste (vv. 9-11) ${ }^{65}$, delle corna (vv. 12-17) e della donna (v. 18) ${ }^{66}$.

64 Secondo E. Corsini, Apocalisse prima e dopo, p. 329 la Bestia dal mare significa la corruzione del potere politico. Quasi tutti i commentatori vi hanno visto e continuano a vedervi un simbolo di Roma e del suo impero. Lautore riconosce quest'interpretazione come "sostanzialemnte esatta". Sulla possibilità di interpretare la Bestia alla luce dell'Anticristo in 2 Ts 2,3-11, cf. P. Ostański, Objawienie Jezusa Chrystusa, p. 284.

65 In 17,9b prosegue la spiegazione dell'angelo e lui dà l'interpretazione delle sette teste

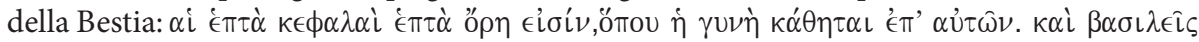
$\dot{\epsilon} \pi \tau \dot{\alpha} \epsilon \dot{i} \sigma \nu$. Le sette teste hanno una doppia spiegazione: sono sette colli e anche sette re. Le sette teste della bestia vengono interpretate in primo luogo come sette monti. Questo primo chiarimento serve soltanto a mostrare che l'immagine si riferisce a Roma, la città dai sette colli, cf. E. Lohse, L'Apocalisse, p. 165. Invece secondo E. Corsini, Apocalisse prima e dopo, p. 444 il termine monte può simbolizzare anche un'entità spirituale opposta a Dio, di orgoglio che si innalza e attenta alla sua sovranità. Secondo alcuni questo simbolo doveva essere corrente nella tradizione giudaica. É anche vero che nella seconda tromba, l'Apocalisse ci descrive la caduta di Satana come quella di "un monte grande, fiammeggiante...scagliato nel mare" $(8,8)$. I sette monti sarebbero dunque il simbolo della pienezza di orgoglio terrestre opposto totalmente a Dio.

Come abbiamo già notato in 17,9, l'angelo dà una doppia spiegazione del simbolismo delle sette teste della bestia. La seconda spiegazione dice che le sette teste sono dei sette re: $\alpha \dot{i}$

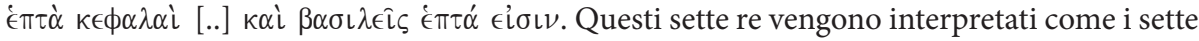
imperatori romani, tenendo presente la prima spiegazione (la città delle sette colli è Roma) e anche il fatto che in Oriente gli imperatori romani erano di solito chiamati re, cf. E. Lohse, L’Apocalisse, pp. 165-166 per un'interpretazione "storicizzante"; anche P. Ostański, Objawienie Jezusa Chrystusa, pp. 285-286. Prendendo però in considerazione il simbolismo del numero sette nell'Apocalisse possiamo interpretare i sette re come immagine della totalità di potenza malefica del mostruoso nemico di Dio. Lordine cronologico preciso non interesserebbe il nostro autore. Secondo E. Corsini, Apocalisse prima e dopo, p. 446 il simbolo delle sette teste sta probabilmente a indicare la dominazione esercitata dalla totalità (numero sette) degli spiriti malvagi sul mondo fisico e umano.

66 Con la spiegazione del personaggio della donna, il programma annunciato dall'angelo nel v. 7 trova compimento. Se questo simbolismo viene per ultimo, lo si deve al fatto che da un lato si trova condizionato da quello della Bestia e della sue teste, e che dall'altro è stato annunciato e preparato a puntino dalle allusioni precedenti (Ap 14,8; 16,19). Ora si capisce ancora più chiaramente che si tratta di Roma. Ma non tutti condividono quest'interpretazione, cf. E. Corsini, Apocalisse prima e dopo, pp. 451-452 identifica la grande prostituta con Gerusalemme. 


\subsection{Le dieci corna sono dieci re (17,12bis.16)}

Prendiamo adesso in esame il testo di 17,12 che spiega direttamente il significato

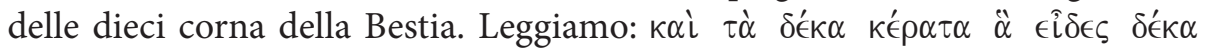

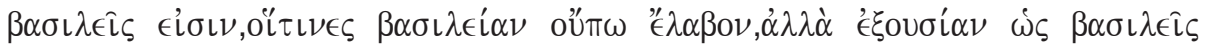

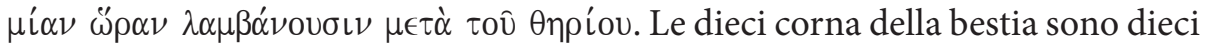
re che non sono entrati ancora in possesso della regalità, ma insieme con la Bestia, avranno potere per un breve tempo.

Siamo all'interpretazione delle dieci corna. Prendendo in considerazione la spiegaziona data prima che le sette teste della Bestia sono i sette re $(17,9)$, secondo alcuni commentatori, si tratterebbe adesso degli stessi sovrani simboleggiati in precedenza dalle teste, e ora contati secondo una lista più completa. Alcuni seguono l'interpretazione "storica" vedendo nei dieci re: i re vassalli di Roma, i Parti, i Barbari oppure satrapi parti, presso i quali si è talora pensato che Nerone abbia trovato rifugio, quando lo si credeva morto. Si aspettava il suo ritorno vendicatore alla testa degli eserciti orientali ${ }^{67}$.

Prima di tutto bisogna notare che l'influsso di Dn 7,24 dove si dice che

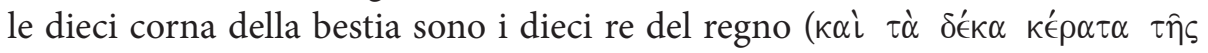

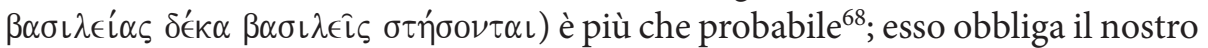
autore a continuare la sua spiegazione profetizzando la venuta futura di dieci re che "ancora non sono entrati in possesso della regalità" (oï $\tau \iota \nu \in \varsigma \beta \alpha \sigma \iota \lambda \in i \alpha \nu$ oü $\pi \omega$ " $\lambda \alpha \beta \beta \nu$ ). Questi sovrani escatologici hanno come caratteristica principale una dedizione totale alla Bestia, cui danno aiuto nella sua ultima manifestazione. Sono quindi le forze che la Bestia può schierare nella battaglia decisiva contro l'Agnello $(17,14)^{69}$. Per questo si devono identificare questi dieci re futuri con i re di tutta la terra che ritroveremo schierati attorno alla Bestia per la battaglia finale. Come ci dice E. Corsini "nelle dieci corna della bestia possiamo vedere, con buona probabilità, rappresentata la serie dei sovrani umani che fanno un uso malvagio del loro potere, mettendosi così oggettivamente al servizio di Satana e in opposizione all'Agnello, cioè a Cristo" ${ }^{70}$. Il nostro testo menziona il numero dei re con la cifra dieci che avendo il valore totalizzante e allo stesso tempo aperto, viene usata per indicare la loro serie indefinita. Le dieci corna significano quindi tutte le forze negative e contrarie a Dio nella storia del mondo ${ }^{71}$. Grazie al simbolismo aritmetico usato lautore sottolinea anche

\footnotetext{
67 Cf. E. Lohse, L’Apocalisse, p. 166; P. Ostański, Objawienie Jezusa Chrystusa, p. 287.

68 Cf. E. Lohse, L'Apocalisse, p. 166.

69 Cf. E. Lupieri, L’Apocalisse di Giovanni, pp. 175-176.

70 Cf. E. Corsini, I numeri nell'Apocalisse, p. 401.

71 Cf. G. Ravasi, Apokalipsa, p. 149.
} 
il fatto che la forza e la potenza dei re della terra che insieme con la Bestia entrano nella battaglia finale contro l'Agnello sono in qualche modo limitate e vengono sconfitte dal Cristo - Agnello nella sua morte e nella sua risurrezione $(19,16)$.

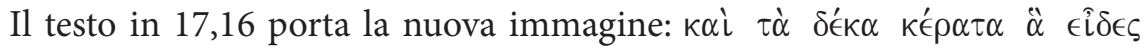

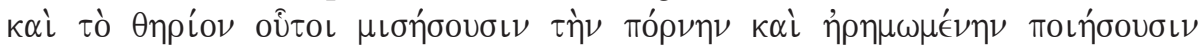

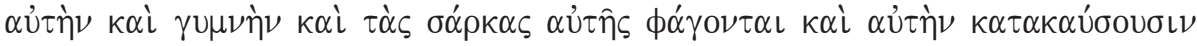
$€ \mathcal{~} \mathcal{V}$ mupí. La Bestia e il suo seguito, cioè i dieci re, devasteranno la prostituta, la spoglieranno e la distruggeranno. La profezia viene formulata in termini tradizionali che ricordano molti testi dell'Antico Testamento. In Ez 23,25-29 la sorte di Gerusalemme è di essere consegnata all'odio dei nemici, che la denudano e l'incendiano. Questo è il castigo della sua prostituzione ${ }^{72}$. Ciò che sembra chiaro e che l'autore vuole esprimere è il fatto che il giudizio di Babilonia diventa il castigo che avrebbe colpito alla radice stessa del male. Paradossalmente "Dio stesso dunque si serve delle potenze sataniche per punire l'empia Babilonia. La capitale del mondo splende ancora nella sua enorme potenza, ma la sua fine spaventosa si avvicina inarrestabilmente" ${ }^{73}$. Il numero dieci delle corna ancora una volta esprime la forza dei poteri malvagi che da una parte sembra essere indefinita e totale, ma rimane sempre limitata in quanto legata al male vinto definitivamente dal Risorto. Ancora una volta riportiamo l'opinione di Corsini: "La raffigurazione della bestia con le sette teste e le dieci corna rappresenta, dunque, il simbolo riassuntivo della stroria umana, in quanto è sotto il segno e il dominio del peccato e di satana. Essa copre con il suo simbolo tutto il tempo dell'attesa, dalla caduta all'avvento di Cristo"74.

\section{Conclusione}

A conclusione del nostro articolo vogliamo, dando uno sguardo retrospettivo al cammino percorso, raccogliere in sintesi alcune osservazioni. Il simbolismo aritmetico occupa uno spazio importante nell'ultimo libro del Nuovo Testamento. Tra i diversi numeri riportati nell'Apocalisse la cifra dieci ricorre 9 volte. Come già negli altri testi biblici dieci è un numero tondo, corrispondente alle dita delle mani che suggerisce abbondanza e grandezza ed esprime una quantità approssimativa. Nell'Apocalisse questo numero viene associato sempre con il demoniaco, particolarmente con la durata della persecuzione $(2,10)$, con

72 P. Ostański, Objawienie Jezusa Chrystusa, p. 289.

73 E. Lohse, L'Apocalisse, p. 167.

74 E. Corsini, I numeri nell'Apocalisse, pp. 448-449. 
le corna $(12,3 ; 13,1 ; 17,3.7 ; 12$ bis.16), con i diademi $(13,1)$ del Drago e della Bestia. Da una parte sia le corna sia i diademi simboleggiano forza e potere straordinari, ma sono dieci, cioè limitati e non comparabili con l'onnipotenza divina delle sette corna dell'Agnello $(5,6)$ e del potere del Cavaliere che "ha sul suo capo molti diademi" $(19,12)$. Possiamo dire insieme con N. Marconi che il significato del numero dieci "è di una pienezza e forza del tutto rilevante, ma inferiore alla completezza"75. Il numero dieci sembra indicare una limitatezza nonostante l'apparenza del contrario che poi essa viene determinata e chiarita dal contesto. E così un tempo che sembra lungo e senza fine, in realtà è un tempo limitato (dieci giorni della persecuzione in 2,10 ), la potenza è limitata (dieci corna del Drago) nonostante l'apparenza minacciosa di forza travolgente, suggerita dal simbolismo teriomorfo $(12,3)$ sia del Drago sia della Bestia. Similmente la regalità della Bestia è limitata $(13,1 ; 17,3)$ anche se questa potenza (corna) della Bestia si incarna nella struttura della storia umana (diademi), ma è sempre la realtà vista nella sua limitatezza (dieci), la forza e la potenza dei re della terra che insieme con la Bestia entrano nella battaglia finale contro l'Agnello sono limitate (17,12bis.16). Possiamo dire che il numero dieci legato sempre nell'Apocalisse con le forze del male esprime la totalità dei poteri contrari a Dio, a Cristo e ai suoi seguaci nella storia del mondo. Ė una potenza enorme e sconvolgente, ma limitata e vinta già dallopera salvifica di Colui che "Signore dei signori e Re dei re" $(17,14$ e 19,16). La cifra dieci esprime simbolicamente quindi il male che è limitato nel tempo e nello spazio. Esso possiede una grande forza e spesso si rivela come un potere distruggente, però non è mai onnipotente.

Così, in rapida sintesi, si presenta il significato simbolico della cifra dieci nel libro dell'Apocalisse. Grazie al collegamento con altri simboli biblici, l'autore attraverso il simbolismo aritmetico esprime le varie idee teologiche. In questo modo il messaggio del libro, nascosto sotto il linguaggio simbolico, diventa più affascinante. É indirizzato all'uomo di ogni tempo e chiede da lui un vero impegno nel decifrare ciò che lo Spirito dice alla Chiesa nel momento concreto della sua storia.

\section{Summary}

The Apocalypse, as the last book of the New Testament, is known of its symbolism, where symbolism of numbers plays an important role amongst other categories. The article addresses the symbolism of number ten in that Book.

75 N. Marconi, Le mille immagini dell'Apocalisse, p. 71. 
Beginning with the Old Testament, number ten symbolizes certain completeness, and often is part of elements' listing of ten. This fact alone shows its symbolic meaning, indicating fullness, greatness and completeness. The New Testament continues that symbolism. In the Apocalypse, number ten appears 9 times, always in the context of opposition to God and to the Son of God. It embraces certain negative aspect of completeness, yet it remains limited.

Letter to the Church in Smyrna, includes the prophecy of persecution, which as being devil's creation will last ten days $(2,10)$, symbolizing a short and limited trial period, after which there will be victory of those faithful to Christ. Further, in 12,3, a ten horned Dragon / Devil appears, symbolizing his great strength and power, but also remaining limited. Likewise, the Beast emerging from the sea, has ten horns and ten diadems $(13,1 ; 17,3.7 ; 12[\mathrm{x} 2] .16)$. Again, it symbolizes Beast's great royal powers (diadems). Further, an angel explains that ten horns represent ten kings $(17,12.16)$. Symbolically speaking, this represents all powers, which throughout history have served the Beast, realizing its goals in opposition to God, Christ, and His followers. Even though, the power of the Beast and its servants seems to be great and limitless, it is in fact limited by the symbolism of number ten, so frequently used in the Beast's description. The Beast gets defeated by the Lamb, having fullness of power, expressed by seven horns $(5,6)$ and being a victorious Rider, with multiple diadems on His head. The powers of the Dragon, the Beast and their followers get defeated and limited by paschal mystery of Jesus Christ.

In conclusion, number ten in the Apocalypse, symbolizes the Evil, which remains limited in time and space. Despite its great powers, it is neither almighty nor limitless. God and the Lamb - resurrected Jesus - rule over the Evil. 